\title{
Characterization of the PANDA MVD Trapezoidal Silicon Strip Sensors and their First Operation in a Proton Beam
}

\author{
Dariusch DEERMANN* \\ Forschungszentrum Jülich \\ E-mail: d.deermannefz-juelich.de \\ Dr. Tobias Stockmanns \\ Forschungszentrum Jülich \\ E-mail: t.stockmannsefz-juelich.de
}

\section{Prof. Dr. James Ritman}

Forschungszentrum Jülich

E-mail: j.ritmanefz-juelich.de

The $\bar{P} A N D A$-experiment will be one of the main experiments at the upcoming Facility for Antiproton and Ion Research (FAIR) at the GSI in Darmstadt. The fixed target experiment will explore $\bar{p}$ p annihilations in the charm mass region with intense, phase space cooled beams with momenta between 1.5 and $15 \mathrm{GeV} / \mathrm{c}$.

The innermost subdetector of $\bar{P} A N D A$ will be the Micro Vertex Detector (MVD) and will consist of silicon strip and pixel detectors. The MVD can be further divided into two sub-structures. A barrel-structure around the vertex and a disc-structure in beam direction with six disks of different size. The last two disks are hybrid disks with trapezoidal strip sensors in the outer layer surrounding a smaller ring of pixel sensors in the inner part. The other disks are made only out of pixel detectors.

The first trapezoidal prototype sensors were produced by CiS ${ }^{\dagger}$ and have a stereo angle of $15^{\circ}$ with 512 strips per sensor side and a strip pitch of $67.5 \mu \mathrm{m}$.

In order to operate and test the prototype sensors, they were chracterized with a probe station as well as with a dedicated testboard. A first beam test was done at COSY with protons of $2.95 \mathrm{GeV} / \mathrm{c}$ and $800 \mathrm{MeV} / \mathrm{c}$ in December 2013 and January 2014.

Technology and Instrumentation in Particle Physics 2014,

2-6 June, 2014

Amsterdam, the Netherlands

\footnotetext{
* Speaker.

${ }^{\dagger}$ Forschungsinstitut für Mikrosensorik und Photovoltaik GmbH

\$Cooler Synchrotron at Forschungszentrum Jülich GmbH
} 


\section{Trapezoidal Sensors}

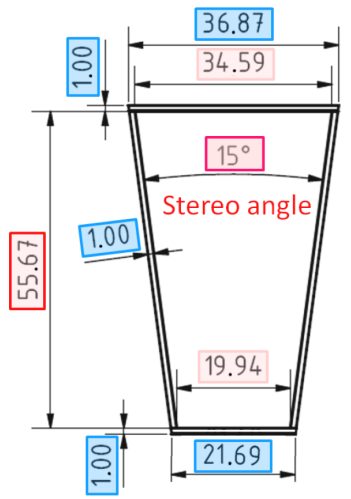

Figure 1: Scheme of the trapezoidal sensor's geometry[1]
Trapezoidal double-sided silicon strip sensors will form the outer ring of the forward discs in the $\bar{P} A N D A$ MVD[1].

Their prototypes have 512 strips per sensor side with a pitch of $67.5 \mu \mathrm{m}$ and a stereo angle of $15^{\circ}$. The strips are parallel to the long edges of the sensor and, therefore, vary in length according to the strips' position. With the bulk material being $n^{-}$-doped, the microstrips are $p^{+}$-doped on the p-side and $n^{+}$-doped on the $\mathrm{n}$-side of the sensor. Strip separation on the sensor's n-side is realized by the p-spray method, which means that inbetween the $n^{+}$-doped strips a $p^{-}$-doped area is implemented. The used biasing technology is punch-through, which leaves a small gap of $n^{-}$-doped bulk material inbetween the bias rings of both sides and the corresponding strips.

Figure 1 shows the sensor's geometry with all lengths given in $\mathrm{mm}$.

\section{Sensor Characterization}

Two methods of characterization have been set up and used. With a probe station it is possible to perform non-destructive measurements on sensors which are meant to be used in a detector afterwards. However, this setup is limited in the number of possible contacts which are realizable with needles. Also, only contacts from one sensor side at a time are accessible as the sensor is lying on the opposing side.

A second method of characterization was realized by designing a dedicated testboard on which a sensor is fixed with glue and all the contacts from both sensor sides are wirebonded to that board. In this situation we cannot use the sensor in another setup afterwards, but there are no limitations to the number of contacts and both sides are accessible at all times. Also, the wirebonds are much smaller than the needles of the probe station and therefore generate less noise contribution.

\subsection{Characterization with the Probe Station}

The probe station as well as the measurement devices can be remotely controlled and a measurement software has been written to perform automated measurement series. The capacitance of a silicon sensor is directly correlated to the width of the depletion zone which acts as the seperating layer between the two electrodes. Therefore, the capacitance will decrease with increasing reverse biasing voltage until full depletion is reached.

In Figure 2(a) the measurement for this behavior is shown and it reveals full depletion at around $115 \mathrm{~V}$.

For single strips the capacitance is also dependent on the strip length which can be seen in Figure 2(b). Since it is not possible to contact all of the 512 strips on one side at the same time on the probe station, the biasing contacts were chosen as alternative measurement points for a sensor side. The punch-through mechanism inbetween the biasing ring and the strips serves as a resistor, which causes a lower measured value compared to the results with the testboard on which the strips can be contacted directly. Due to the contact by needles, the measurements have higher noise. This 

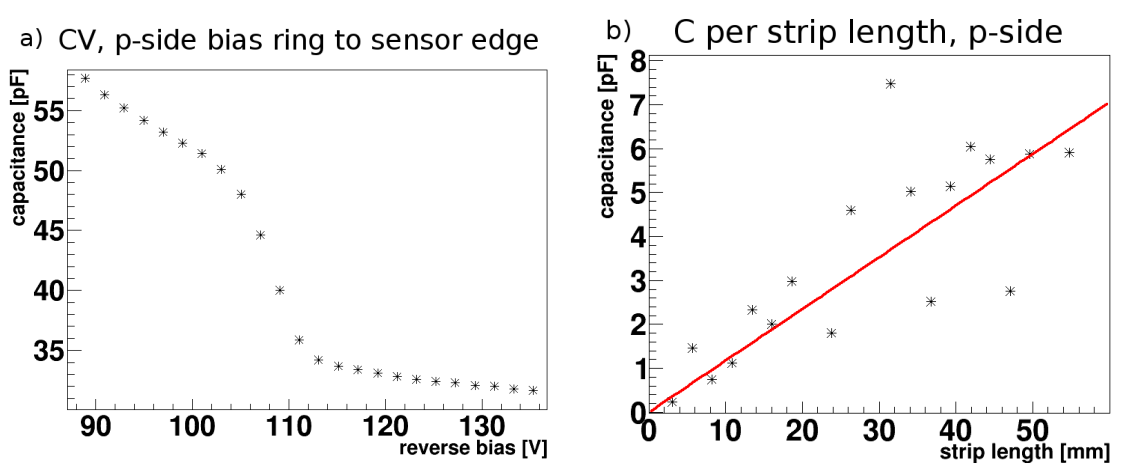

Figure 2: a) Capacitance measured for different biasing voltages; b) Capacitance for different strip lengths

is especially obvious in the plot of the relation between capacitance and strip length, where we deal with very small capacitances. But the tendency of increasing capacitance with increasing strip length is still visible.

\subsection{Characterization with the Dedicated Testboard}

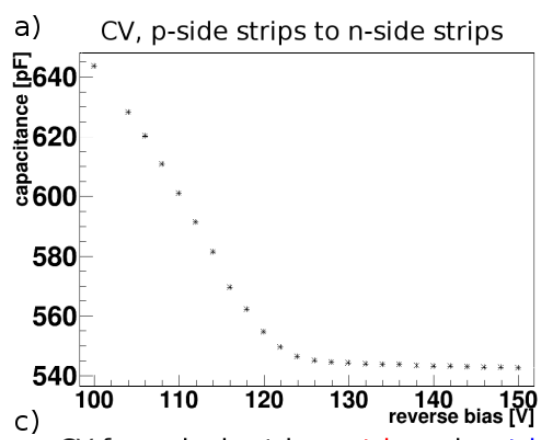

b) $\mathrm{C}$ per strip length, $\mathrm{p}$-side and $\mathrm{n}$-side
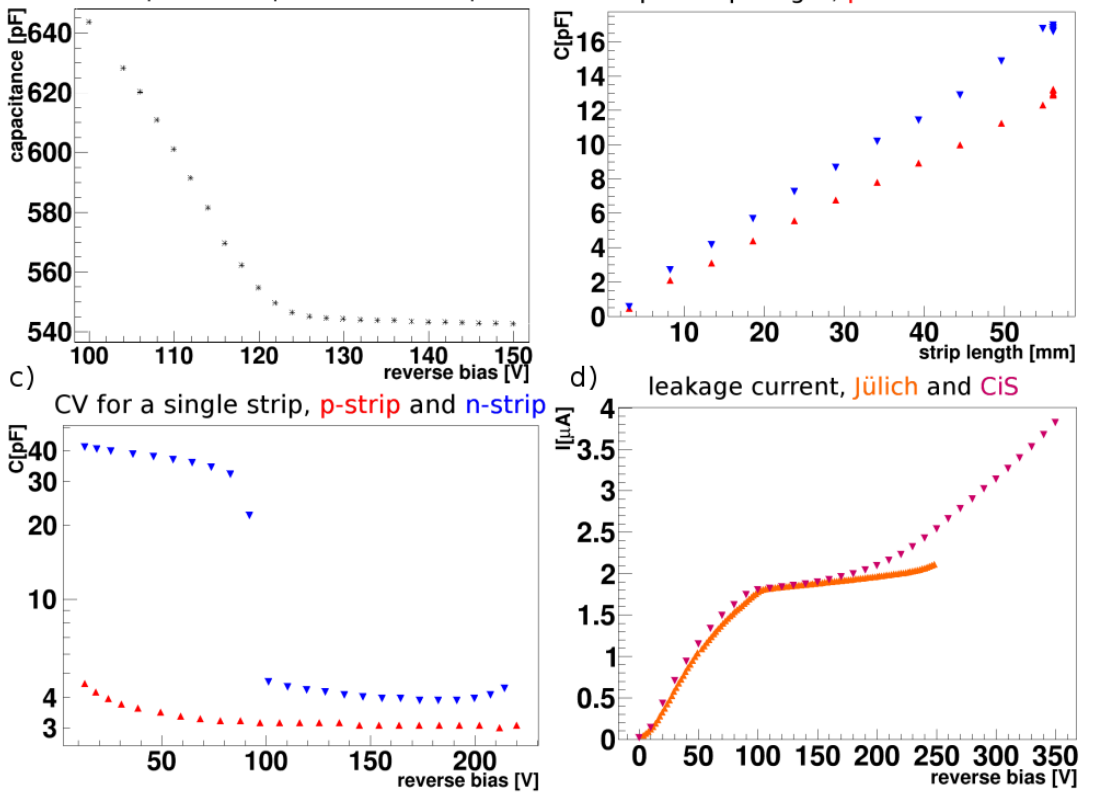

Figure 3: a) CV measurement of the sensor; b) Capacitance for different strip lengths; c) CV measurement for a single strip; d) Leakage current of the sensor

Since all sensor strips are wire bonded to the testboard, there is no need to take the biasing contacts as alternative sensor side contacts. Because of the direct contact to the strips the systematic error is lower compared to the probe station measurements. Also, the noise contribution is lower due to the better contact provided by the small wire bonds in comparison to the big needles on the probe station. 
As seen in Figure 3 the measured full depletion voltage for this sensor is at about $125 \mathrm{~V}$. The capacitance to strip length relation is now much clearer and can be measured for both sensor sides individually. It is $\sim 0.29 \mathrm{pF} / \mathrm{mm}$ for the $\mathrm{n}$-side and $\sim 0.22 \mathrm{pF} / \mathrm{mm}$ for the $\mathrm{p}$-side.

The difference between $\mathrm{p}$ - and $\mathrm{n}$-side becomes visible when looking at the capacitance of a single strip. The p-side strip behaves just as the whole sensor scaled down to a lower capacitance. But the n-side strip shows a big capacitance at low biasing voltages and has a sharp drop at about $90 \mathrm{~V}$.

This is because the $n^{+}$-doped strips are interconnected to each other via the $n^{-}$-doped material until the depletion zone grows big enough to touch the seperating p-spray area.

As for the quality assurance of the sensor a comparison has been made between the manufacturers specification of the leakage current and the leakage current measured in our laboratory. The result can be see in Figure 3(d) and it shows an even lower leakage current at high biasing voltages compared to the specification from CiS. The specification by CiS stated an operating temperature of $20^{\circ} \mathrm{C}$ while our laboratory's temperature was at $19.5^{\circ} \mathrm{C}$ at time of measurement.

\section{Beam Test}

The prototype sensor has been tested in a proton beam at COSY with beam momenta of $0.8 \mathrm{GeV} / \mathrm{c}$ and $2.95 \mathrm{GeV} / \mathrm{c}$. The p-side of the sensor showed many noise entries, which deposited a low cluster charge and are easily separable from the signal entries as seen in Figure 4(b) ${ }^{1}$. Also, the correlation between $\mathrm{p}$ - and n-side showed a linear behavior with some noise and ghost entries, which can be reduced by a cut, too. Figure 4(a) shows a hitmap of the sensor after cuts on low energy clusters and the p- to n-side correlation have been applied. The beamspot in that hitmap is clearly visible, though the upper left corner is missing due to one broken frontend. Taking the charge distribution between the strips of a cluster into account, can enhance the spatial resolution. At the peak in the ADC distribution the p-side showed $15.4 \%$ two strip clusters and the n-side $45.3 \%$. For the two strip clusters the $\eta$ method can be applied. $\eta$ is defined as $\eta:=\frac{q_{r}}{q_{r}+q_{l}}$, with $q_{r}$ being the charge of the right strip and $q_{l}$ the charge of the left strip. After measuring the $\eta$ distribution $N(\eta)$ for the sensor the position of a hit can be reconstructed by the formula $X(\eta)=x_{l}+\frac{\int_{0}^{\eta} N\left(\eta^{\prime}\right) d \eta^{\prime}}{\int_{0}^{1} N\left(\eta^{\prime}\right) d \eta^{\prime}}[2]$. In that formula $x_{l}$ is the position of the left strip. The $\eta$ distribution may differ for different energy regions, so it is advisable to plot the $\eta$ distribution in a 2D diagram by entries per $\eta$ value and cluster energy as seen in Figure 4(d).

\section{Summary}

With the dedicated testboard a precise characterization of a trapezoidal sensor was performed. This characterization gives insight into the sensors technological behavior and provides a test setup to check the delivered quality of the sensors. Nevertheless, it is also necessary to perform nondestructive quality assurance tests of the sensors before installation in the detector. Therefore, with the probe station a second test system has been set up, which is slightly less precise but nondestructive. For both systems a software has been written to perform automated measurements. The first beam test of the trapezoidal sensor showed a clear hitmap after noise and correlation cuts

\footnotetext{
${ }^{1}$ The average Signal to Noise Ratio was $\sim 29$ for the p-side
} 

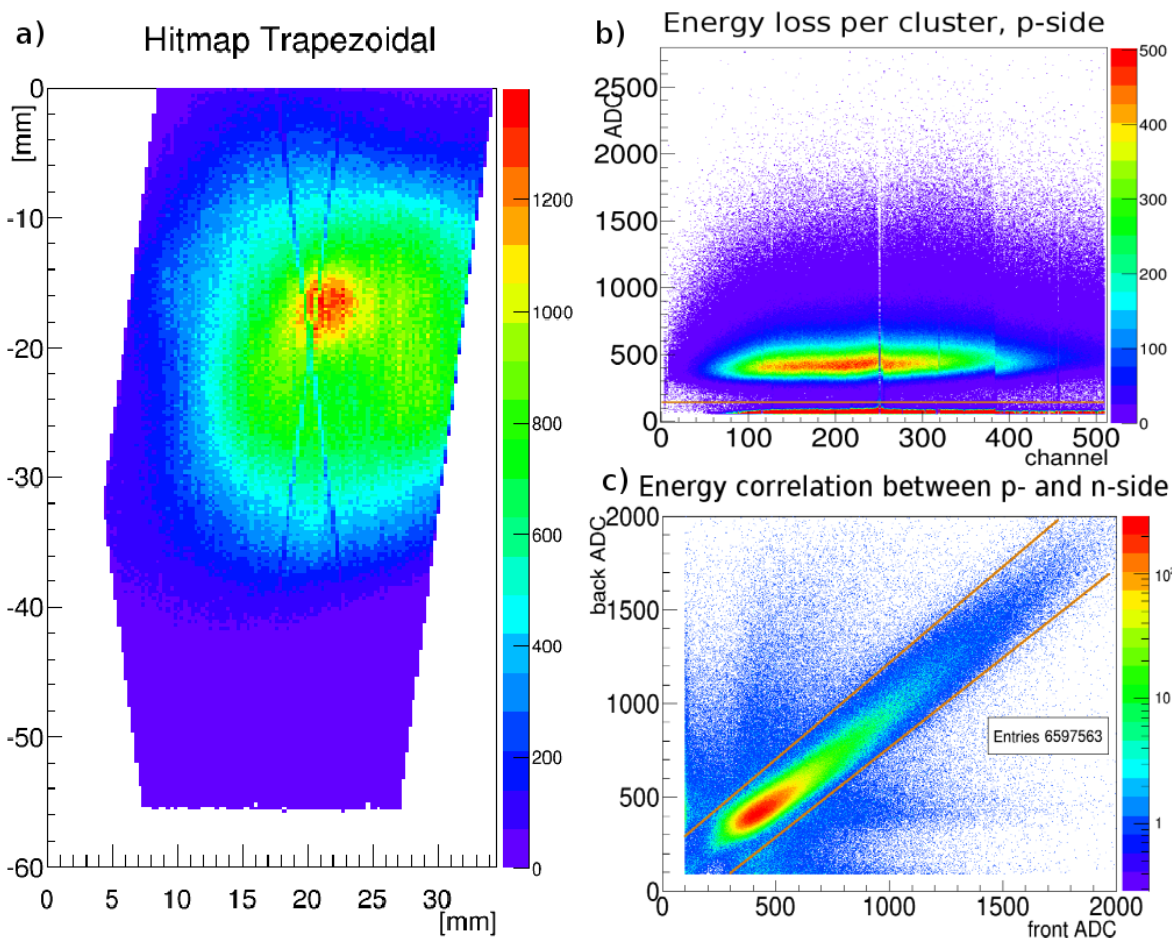

c) Energy correlation between $\mathrm{p}$ - and $\mathrm{n}$-side

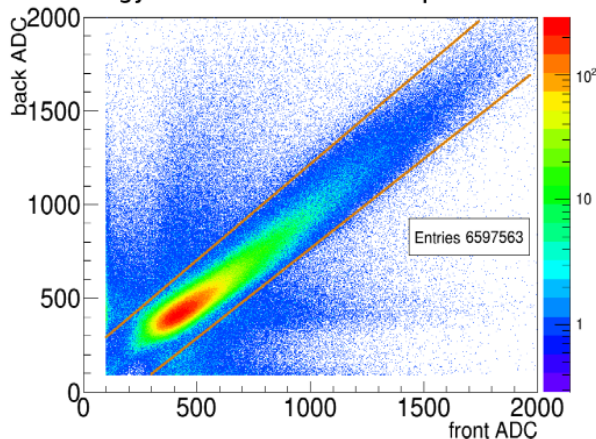

d)

Eta distribution for all Energies, top

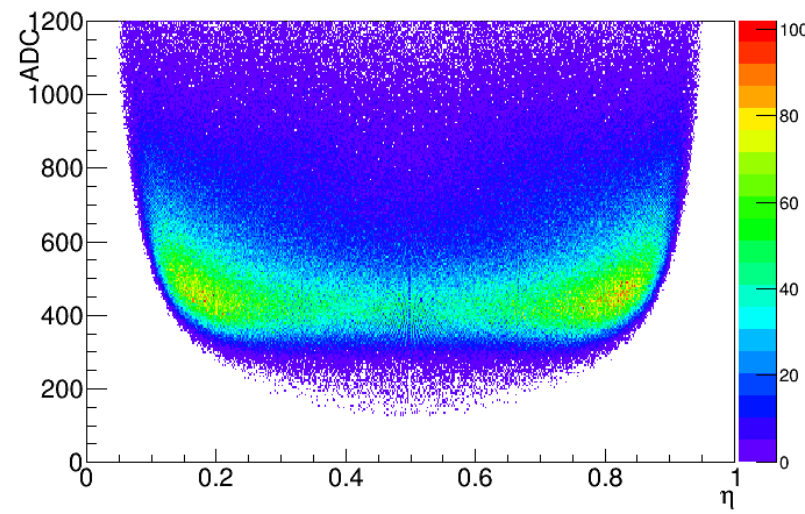

Figure 4: a)Hitmap of the sensor; b)Cluster charge on the p-side; c)Correlation between p- and n-side of the sensor; d) p-side $\eta$ distribution; Cuts in b) and c) are indicated by orange lines

had been applied. Also, cluster charge distribution was present and can be used to enhance the spatial resolution.

\section{References}

[1] Technical Design Report for the: PANDA Micro Vertex Detector, arXiv:1207.6581

[2] E. Belau et al., "Charge collection in silicon strip detectors", Nuclear Instruments and Methods in Physics Research, vol. 214, no. 2-3, pp. 253-260, 1983. 